\title{
INQUÉRITO CANINO EM FOCO RECENTE DE LEISHMANIOSE TEGUMENTAR NO MUNICÍPIO DE SABARÁ, REGIÃO METROPOLITANA DE BELO HORIZONTE
}

\author{
Valêria Maria Azeredo Passos, Ana Cristina Andrade, Eduardo Sérgio Silva, \\ Elizabeth Maria Figueiredo e Alda Lima Falcão
}

\begin{abstract}
Em 1992, foi obseriada lesão cutânea por Leishmania em um cão do município de Sabarā, Minas Gerais, onde já baviam sido registrados casos bumanos de leisbmaniose tegumentar. O parasita foi caracterizado como pertencente ao subgênero Viannia, ao qual pertence a Leishmania braziliensis, principal espécie encontrada na regiâo sudeste do Brasil. Com o objetito de determinar o papel do cão no ciclo de transmissão da doença, foi realizado um inquérito canino na área. Foram examinados 631 cães, sendo a soroprevalência para leishmaniose igual a 3,2\%. Foi observada a proximidade ou presença no mesmo domicítio de cães e pessoas doentes. Este aspecto fala a favor da transmissão domiciliar ou peridomiciliar; com o cãa infectado podendo atuar como fator de risco nesta ârea periurbana. Entretanto, a baixa soroprevalência encontrada deve-se provavelmente ao pequeno papel deste animal na transmissão da doença neste foco recente da doença.
\end{abstract}

Palauras-chaves: Leishmaniose tegumentar. Inquérito canino. Leishmania (Viannia) sp.

A leishmaniose tegumentar americana (LTA) é classicamente definida como uma zoonose de áreas de mata primária. O estudo de seus reservatórios silvestres no Brasil se inicia com as observações de Brumpt e Pedroso 5 , quando foram encontradas lesões clinicamente sugestivas em duas cutias (Dasyprocta). Desde então, os animais silvestres são considerados os principais reservatórios da doença em várias áreas endêmicas do país ${ }^{+}{ }_{18} 27$. Entretanto, a doença vem sendo observada em áreas com ambientes já bastante alterados, onde os animais silvestres são escassos ou mesmo ausentes. Já em 1960, ao estudar os animais silvestres de área de colonização recente onde ocorrera casos humanos de LTA, Forattini discutia a possibilidade de adaptação do parasita a animais domésticos, quando o ambiente se torna densamente povoado. Nas últimas três

\footnotetext{
Laboratório de Epidemiologia e Antropologia Médica, Laboratório de Leishmanioses, Centro de Pesquisas René Rachou/Fundação Oswaldo Cruz, Belo Horizonte, MG.

Órgão financiador: Fundação de Amparo à Pesquisa de Minas Gerais/FAPEMIG

Endereço para correspondência: $\mathrm{Dr}^{\text {a }}$ Valéria Maria de Azeredo Passos. Centro de Pesquisas René Rachou. Av. Augusto de Lima 1715, 30190-002 Belo Horizonte, MG. Tel: (031) 295-3566, Fax: (031) 295-3115.

Recebido para publicação em 12/05/95.
}

décadas, a LTA vem sendo observada em zonas rurais e na periferia de grandes centros urbanos, onde o cão tem sido encontrado infectado e muitas vezes relacionado à ocorrência de surtos em casos humanos ${ }^{1392430}$.

Em outubro de 1992, foi encontrado um cão com uma lesão característica de LTA proveniente do bairro Santo Antônio de Roça Grande, município de Sabará, na Região Metropolitana de Belo Horizonte (RMBH). A reação de imunofluorescência indireta para leishmaniose deste animal foi positiva e foram observadas amastigotas em esfregaço de pele corado pelo Giemsa. O parasita foi caracterizado como pertencente ao subgênero Viannia, através da detecção pela reação de polimerização em cadeia utilizando-se oligonucleotídeos flanqueadores da região conservada de minicírculo de kDNA e tipagem por sonda molecular radioativa de minicírculo clonado de kDNA de L. panamensis ${ }^{21}$.

Neste município, desde 1987, já se observavam casos humanos de LTA, que acomete indistintamente adultos e crianças de ambos os sexos, com atividades profissionais ou de lazer não relacionadas com áreas de mata $^{19}$, sendo que nos anos de 1992 e 1993 foram notificados 17 casos humanos do bairro Santo Antônio de Roça Grande (K Magalhães: comunicação pessoal, 1995) 
Passos VMA, Andrade AC, Silva ES, Figueiredo EM, Falcão AL. Inquérito canino em foco recente de leisbmaniose tegumentar no município de Sabará, região metropolitana de Belo Horizonte. Retista da Sociedade Brasileira de Medicina Tropical 29:323-329, jul-ago, 1996.

Com o objetivo de determinar a prevalência da infecção canina nesta localidade, optou-se pela realização de um inquérito canino na área, visando conhecer o papel deste animal doméstico no ciclo de transmisão da doença.

\section{MATERIAL E MÉTODOS}

O bairro Santo Antônio das Roça Grande pertence ao município de Sabará, a cerca de $20 \mathrm{~km}$ de Belo Horizonte, situando-se a 19.53'59" lat S e 43\%49'06" lat W, com altitude em torno de $705 \mathrm{~m}$ do nivel do mar. A população total do município estimada para 1989 é de 88.980 habitantes, sendo a população urbana de 86.962 e a rural de 2.018 habitantes ${ }^{25}$. Caracteriza-se como região de colonização antiga, sem áreas de lavoura, com escassas matas secundárias restritas ao topo dos morros. Muitas casas possuem árvores frutíferas no peridomicílio, principalmente mangueiras e bananeiras. O município não é endêmico para doença de Chagas, mas há cerca de três anos vêm-se registrando casos de calazar canino ${ }^{12} 19$.

De janeiro a março de 1993 , todas as casas do bairro foram mapeadas. Foram obtidas informações sobre o sexo, idade, procedência, tempo de residência na área e atividades fora da área dos cães residentes no bairro.

Todos os cães residentes nas casas previamente mapeadas foram examinados, procurando-se detectar lesôes tegumentares. Foram consideradas lesões suspeitas as úlceras com diâmetro igual ou maior que $0,5 \mathrm{~cm}$ em qualquer área do corpo e nódulos em áreas de pouco pelo; alterações cicatriciais, manchas, escoriaçôes e peladeiras foram consideradas não-suspeitas.

O sangue dos animais foi coletado em Vacutainer, as amostras de soro congeladas a $-20^{\circ} \mathrm{C}$ e posteriormente submetidas à reação de imunofluorescência indireta (RIFI) para leishmaniose, utilizando-se antígeno de $L$. (L.) amazonensis. Todo soro com título igual ou superior a 1:40 foi considerado positivo ${ }^{1 ?}$.
$\mathrm{Na}$ análise estatística foram utilizados os testes do qui-quadrado, o teste exato de Fisher e o teste $T$ de Student para comparação das características dos cães com sorologia positiva e negativa. $O$ nível de significância considerado foi de $5 \%(\alpha=0,05)^{28}$.

\section{RESULTADOS}

Foram mapeadas 723 casas, com um total de 631 cães em 441 casas $(x=1,43), 326$ machos e 301 fêmeas, com média de idade de $34,9 \pm 18,7$ meses, sendo $554 \quad(87,8 \%)$ procedentes da área, $68(10,8 \%)$ de outros municípios da RMBH e $9(1,4 \%)$ de outras regiões do estado. A maioria dos cães $(98,4 \%)$ era mantida apenas no peridomicílio para guarda das casas e apenas $10(1,6 \%)$ cães tinham alguma atividade fora da região.

Foram encontrados 52 cães com lesões cutâneas suspeitas; um nódulo no pescoço e 51 lesōes ulceradas incaracterísticas, sendo 28 na região de cabeça e pescoço, 21 nas patas e duas no tronco. Vinte e cinco cães apresentavam queda de pelos local ou generalizada.

Foram realizadas $617(97,8 \%)$ RIFI, sendo $20(3,2 \%)$ positivas, 11 com título igual a $1: 40$ e nove com título igual a 1:80. Dos 20 cães com sorologia positiva, 15 eram machos e 5 fêmeas, com média de idade igual a 24,4 meses, variando de 3 a 96 meses. Apenas um cão mantinha atividades fora do bairro, 18 cães eram procedentes e residentes no bairro e 2 cães eram procedentes de Betim, MG, mas já residiam na área há mais de um ano. Nenhum cão com sorologia positiva apresentava lesões sugestivas de LTA à época da realização do inquérito.

Não foram encontradas associaçôes estatisticamente significativas entre sorologia positiva e o sexo $\left(x^{2}\right.$ Yates $\left.=3,58, p=0,06\right)$, idade $(\mathrm{t}=1,54, \mathrm{p}=0,12)$, procedência (Fisher, $p=0,55)$, tempo de residência na área $(\mathrm{t}=1,08, \mathrm{p}=0,28)$, atividades fora $\mathrm{da}$ área (Fisher, $p=0,25$ ) ou presença de lesões cutâneas (Fisher, $\mathrm{p}=0,47)$ (Tabela 1).

Tabela 1- Características dos cāes examinados no bairro Santo Antônio de Roça Grande. Sabará, Minas Gerais, em 1993.

\begin{tabular}{|c|c|c|c|}
\hline Variáveis & $\begin{array}{l}\text { Sorologia positiva para } \\
\text { leishmaniose }\end{array}$ & $\begin{array}{l}\text { Sorologia negativa para } \\
\text { leishmaniose }\end{array}$ & $\begin{array}{c}\text { Odds-Ratio }(\mathrm{OR})(\overline{\mathrm{IC}}=95 \%) \\
\text { ou teste } \mathrm{t} \text { de Student }\end{array}$ \\
\hline Sexo & $M-15 \mathrm{~F}-5$ & $M-311 \mathrm{~F}-296$ & $\mathrm{OR}=2,89(0,97-9,22)$ \\
\hline Idade (média em meses) & $24,40 \pm 22,88$ & $33,85 \pm 27,10$ & $\mathrm{t}=1.54 \mathrm{p}=0,12$ \\
\hline Procedência & RMBH-18 Oa-2 & $\mathrm{RMBH}-518 \mathrm{Oa}-72$ & $O R=0,80(0,12-3,73)$ \\
\hline Tempo de residência na área ( $\mathrm{em}$ meses) & $24,10 \pm 23,14$ & $30.57 \pm 26.49$ & {$[=1,08 \mathrm{p}=0,28$} \\
\hline Atividades fora da área & S-2 N- 18 & S- $8 \mathrm{~N}-570$ & $O R=0,25(0,03-5,76)$ \\
\hline Presença de lesōes cutâneas sugestivas de leishmaniose & S-0 N- 20 & $\mathrm{~S}-52 \mathrm{~N}-537$ & $\mathrm{OR}=0,70(0,11-3,25)$ \\
\hline
\end{tabular}


Passos VMA, Andrade AC, Silua ES, Figueiredo EM, Falcão AL. Inquêrito canino em foco recente de leishmaniose tegumentar no municipio de Sabará, região metropolitana de Belo Horizonte. Revista da Sociedade Brasileira de Medicina Tropical 29:323-329, jul-ago, 1996.

Quando a residência dos cães soropositivos e dos casos humanos ocorridos em 1992 e 1993 foram mapeadas (Figura 1), observou-se que 6 cães residiam a mais de $500 \mathrm{~m}$ dos casos humanos, 10 cães residiam a pelo menos $200 \mathrm{~m}$ e 3 cães residiam a cerca de $500 \mathrm{~m}$ de casos humanos. Um cão foi encontrado na mesma residência de 2 casos humanos, ambos com idade superior a 70 anos e que não saíam da região há pelo menos 5 anos.

\section{DISCUSSÃO}

A LTA, antes restrita a áreas rurais, agora se faz presente em centros urbanos, num processo de urbanização da doença. A caracterização da transmissão da LTA como urbana vincula-se ao conceito de cidade, entendido como "complexo demográfico formado, social e economicamente, por importante concentração populacional não agrícola; i.e.; dedicada a atividades de caráter mercantil, industrial, financeiro e cultural" ${ }^{10}$. No entanto, é flagrante a existência de diferentes graus de urbanizaçâo. Somarriba e cols ${ }^{29}$ preconizam urbano não mais como um conceito geográfico-espacial, mas sim como um local de excelência de reprodução capitalista, onde os trabalhadores de baixa renda são obrigados a residir em áreas cada vez mais periféricas aos centros urbanos, sofrendo a exclusão tanto dos bens de consumo individuais como dos bens coletivos. As áreas de Sabará onde ocorre a LTA são muito mais periurbanas do que realmente urbanas: compostas de população de baixa renda, não possuem coleta de lixo ou sistema de esgotos ${ }^{19}$.

Desde os achados de infecção natural em cães por Pedroso ${ }^{23}$, as investigações sobre reservatórios domésticos da LTA no Brasil se concentram principalmente no estudo deste animal, o que leva à impressão de ser o câo o animal mais freqüentemente infectado. Entretanto, outros animais domésticos já foram encontrados infectados, como equinos ${ }^{1}$, suínos ${ }^{4}$ e felinos ${ }^{22}$. $\mathrm{Na}$ área estudada, não foram investigadas lesôes em outros animais, como equinos e suínos, que estavam presentes em pequeno número.

As lesões leishmanióticas em cães apresentam alternância de períodos de cura e recidiva espontâneas ${ }^{921}$, o que pode explicar a ausência, em nosso estudo, de cães com úlceras sugestivas quando da realização do inquérito. Além disso, em áreas de transmissão de $L$. braziliensis, a prevalência de cães com testes sorológicos e intradérmicos é maior que a prevalência de animais portadores de úlceras cutâneas ${ }^{16}$.

Apesar de não ter sido possível o exame parasitológico dos casos soropositivos, optouse pelo sacrifício dos animais como uma tentativa de controle da doença, dada a alta sensibilidade e especificidade da sorologia ${ }^{62} \mathrm{e}$ ao resultado inconclusivo ou ineficaz do tratamento de cães doentes com o antimonial pentavalente ${ }^{24} 26$.

A prevalência de cães com sorologia positiva na área é baixa, semelhante à encontrada em área rural da Bahia, onde entretanto um maior número de cães foi encontrado com lesões ativas da doença ${ }^{3}$. Em áreas periurbanas onde a doença já se instalou há mais tempo, a prevalência é duas ou três vezes maior?

O baixo percentual de cães procedentes de outras regiôes, ou utilizados em atividades fora da área, fala contra a penetração da doença através de animais domésticos trazidos de outras áreas endêmicas do estado. Entretanto, por tratar-se de um estudo transversal, não podemos afirmar que a migração de pessoas e animais de outras áreas endêmicas do estado e do país não tenha influído no aparecimento da LTA na região.

A presença no mesmo domicílio de cães e pessoas doentes é um aspecto que fala a favor da transmissão em ambiente domiciliat ou peridomicilar ${ }^{29}$. Além disso, a proximidade dos casos humanos e caninos sugere que este animal possa ser um fator de risco para a infecção em áreas periurbanas.

No primeiro cão encontrado na área com lesão sugestiva de LTA foi caracterizado parasita do subgênero Viannia, ao qual pertence $L$. braziliensis, o principal parasita encontrado na região sudeste do país. A caracterização bioquímica e isoenzimática de $L$. braziliensis em infecçōes naturais de cães ${ }^{13: 9132+30}$ se dá principalmente em áreas de colonização antiga das regiões sudeste e nordeste do país. A predominância desta espécie, cujo reservatório animal ainda é desconhecido, infectando animais domésticos em áreas onde a LTA se dá em ambientes já bastante alterados pelo homem, reforça o papel do cão como um reservatório doméstico recente, adaptado às novas condições ecológicas.

Em levantamento epidemiológico, em área de leishmaniose, verificou-se que a RIFI em 
Passos VMA, Andrade AC, Silva ES, Figueiredo EM, Falcão AL. Inquêrito canino em faco recente de leishmaniose tegumentar no município de Sabará, região metropolitana de Belo Horizonte. Retista da Sociedade Brasileira de Medicina Tropical 29:323-329, jul-ago, 1996.



Figura 1- Desenho esquemático da ârea estudada, assinalados os casos bumanos e caninos de leishmaniose tegumentar. 
Passos VMA, Andrade AC, Silva ES, Figueiredo EM, Falcão AL. Inquérito canino em foco recente de leisbmaniose tegumentar no municipio de Sabará, região metropolitana de Belo Horizonte. Revista da Sociedade Brasileira de Medicina Tropical 29:323-329, jul-ago, 1996.

eluatos de sangue canino fornece reações cruzadas em cães infectados com L. braziliensis e L. chagast ${ }^{\text {b }}$. Novos esforços de caracterização de cepas provenientes de animais domésticos da RMBH sâo necessários para se chegar a alguma conclusão da espécie prevalente na região.

A discussão do papel do cão na epidemiologia da LTA é ainda controversa. Alguns autores o consideram apenas um hospedeiro acidental ${ }^{1+15}$. Já outros autores ${ }^{72+}$ avaliam que o papel do cão como elo de ligação da doença dos animais silvestres para o homem no ambiente domiciliar necessita de maiores investigações. A LTA em área rural do Espírito Santo é considerada uma zoonose mantida por cães domésticos ${ }^{26}$. A baixa prevalência de cães com sorologia positiva na área de Sabará deve-se provavelmente ao seu pequeno papel neste foco recente de LTA, se bem que o cão possa estar atuando como um elo de ligação entre os ambientes silvestre e o periurbano.

\section{SUMMARY}

In 1992, a dog naturally infected with Leisbmania uas found in a periurban area of Sabara, State of Minas Gerais, where buman cutaneous leishmaniasis bad been previously described. The parasite was classified as Leishmania, subgenus Viannia, which $\mathrm{L}$. braziliensis, the main species of parasite present in the southeast Brazil, also belongs. In order to assess the importance of the dog in the transmission cycle of the disease, a canine survey was undertaken. Six bundred thirtyone dogs were examined and the prevalence of seropositive dogs for crude Leishmania amazonensis antigen was $3.2 \%$. The presence of infected people and seropositive dogs either near or in the same bouse was observed. This fact suggests some transmission in the domiciliar environment, with the dogs being a risk factor for buman infection in that periurban area. In the other band, the low percentage of seropositive dogs points towards a secondary importance of these animals in the transmission of Leishmaniasis in that recent focus of the disease.

Key-words: Cutaneous leishmaniais. Canine survey. Leishmania (Viannia) sp.

\section{AGRADECIMENTOS}

Ao Dr. Octavio Fernandes e Dr. Wim Degrave, do Depto de Bioquímica e Biologia Molecular do Instituto Oswaldo Cruz, pela colaboração na caracterização molecular do parasita. Aos guardas da Fundação Nacional de Saúde pela colaboração no inquérito canino e ao Sr. Rubens Rezende pelo desenho da área.

\section{REFERÊNCIAS BIBLIOGRÁFICAS}

1. Aguillar CM, Rangel EF, Grimaldi $F^{\circ} \mathrm{G}$, Momem $\mathrm{H}$. Human, canine and equine leishmaniasis caused by Leishmania brasiliensis brasiliensis in an endemic area in the State of Rio de Janeiro. Memórias do Instituto Oswaldo Cruz 82:143, 1987.

2. Araújo Filho NA. Epidemiologia de leishmaniose tegumentar americana na Tha Grande, Rio de Janeiro. Estudo sobre a infecção humana, reservatórios e transmissores. Tese de mestrado, Universidade Federal do Rio de Janeiro, Rio de Janeiro, RJ, 1978.

3. Barreto AC, Cuba-Cuba C, Vexenat JA, Rosa AC, Marsden PD, Magalhães AV. Características epidemiológicas da leishmaniose tegumentar americana em uma região endêmica do Estado da Bahia. II Leishmaniose canina. Revista da Sociedade Brasileira de Medicina Tropical 17:59. 65, 1984

4. Brazill RP, Nascimento MDSB, Macau RP. Infecção natural do porco (Sus scrofa) por Leishmania em foco recente de leishmaniose tegumentar na tha de São Luís, Maranhāo. Memórias do Instituto Oswaldo Cruz 82:145, 1987.

5. Brumpt E, Pedroso AM. Pesquisas epidemiológicas sobre leishmaniose tegumentar das florestas no Estado de São Paulo (Brasil). Anais Paulistas de Medicina e Cirurgia 1:97-136, 1913.

6. Costa CA, Genaro O, Lana M, Magalhães PA, Dias M, Michalick MSM, Melo MN, Costa RT, MagalhãesRocha NM, Mayrink W. Leishmaniose visceral canina: avaliação da metodologia sorológica utilizada em inquéritos epidemiológicos. Revista da Sociedade Brasileira de Medicina Tropical 24:2125, 1991.

7. Coutinho SG, Nunes MP, Marzochi MCA, Tramontano N. A survey for american cutaneous leishmaniasis among 1,342 dogs from areas in Rio de Janeiro (Brazil) where human diseases occur. Memórias do Instituto Oswaldo Cruz 80:17-22, 1985.

8. Dias M. Leishmaniose tegumentar Americana na Zona do Rio Doce, Minas Gerais. Aspectos da doença no homem e estudo de reservatórios.Tese 
Passos VMA, Andrade AC, Silua ES, Figueiredo EM, Falcão AL. Inquérito canino em foco recente de leisbmaniose tegumentar no município de Sabará, região metropolitana de Belo Horizonte. Retista da Sociedade Brasileira de Medicina Tropical 29:323-329, jul-ago, 1996.

de doutourado, Universidade Federal de Minas Gerais, Belo Horizonte, MG, 1982.

9. Falqueto A, Coura JR, Barros GC, Grimaldi $F^{\circ}$ G, Sessa PA, Carias VRD, Jesus AC, Alencar JTA. Participação do cão no ciclo de transmissão da leishmaniose tegumentar no município de Viana, estado do Espirito Santo, Brasil. Memórias do Instituto Oswaldo Cruz 81:155-163, 1986.

10. Ferreira $A B H$. Novo Dicionário da Língua Portuguesa, Rio de Janeiro, Nova Fronteira, 1989.

11. Forattini OP. Sobre os reservatórios naturais da leishmaniose tegumentar americana. Revista do Instituto de Medicina Tropical de São Paulo 2:195-203, 1960.

12. Genaro O, Costa CA, Williams I, Silva JE, Rocha NM, Lima SL, Mayrink W. Ocorrência de calazar em área urbana da Grande Belo Horizonte, Minas Gerais. Revista da Sociedade Brasileira de Medicina Tropical 23:121, 1990.

13. Hermeto MV, Vieira-Dias D, Genaro O, RotondoSilva A, Costa CA, Toledo VPCP. Michalick MSM, Williams P, Mayrink W. Outbreak of Cutaneous Leishmaniasis in the Rio Doce Valey, Minas Gerais, Brazil. Memórias do Instituto Oswaldo Cruz 89:519-521, 1984.

14. Lainson R, Shaw JJ. Leishmaniasis in Brazil. V. Studies on the epidemiology of cutaneous leishmaniasis in Mato Grosso State, and observations on two distinct of Leishmania isolated from man and forest animals. Transactions of the Royal Society of Tropical Medicine and Hygiene 64:654-667, 1970.

15. Le Pont F, Mollinedo S, Mouchet J, Desjeux P. Leishmaniose en Bolivie. IV - Le chien dans les cycles des leishmanioses on Bolivie. Memórias do Instituto Oswaldo Cruz 84:417-421, 1989.

16. Marzochi MCA, Barbosa-Santos EGO. Evaluation of a skin test on the canine mucocutaneous leishmaniasis diagnosis. Memórias do Instituto Oswaldo Cruz 83:391-392, 1988.

17. Marzochi MCA, Coutinho SG, Sabroza PC, Souza WJS. Reação de imunofluorescência indireta e intradermoreação para leishmaniose tegumentar americana em moradores na área de Jacarepaguá (Rio de Janeiro). Estudo comparativo dos resultados observados em 1974 e 1978. Revista do Instituto de Medicina Tropical de São Paulo 22:149-155, 1980.

18. Nery-Guimarães F, Azevedo M, Damasceno R. Leishmaniose Tegumentar- zoonose de roedores silvestres na Amazônia. O Hospital 70:387-395, 1966.
19. Passos VMA, Falcão AL, Marzochi MCA, Gontijo CMF, Dias ES, Barbosa-Santos EGO, Guerra HL, Katz N. Epidemiologic aspects of american cutaneous leishmaniasis in a periurban area of the Metropolitan Region of Belo Horizonte. Minas Gerais State, Brazil. Memórias do Instituto Oswaldo Cruz 88:103-110, 1993.

20. Passos VMA, Falqueto A, Gontijo CMIF. Falcão AL. leishmaniose visceral canina em área urbana do município de Sabará, região metropolitana de Belo Horizonte, Minas Gerais. In: Resumos do XXVIII Congresso da Sociedade Brasileira de Medicina Tropical, Belém p. ${ }^{\top 1} 1992$.

21. Passos VMA, Fernandes O. Andrade AC. Gontijo CMF, Degrave W. Detecção e tipagem de Leishmania da região metropolitãni de Belo Horizonte através da reação de polimerização em cadeia e da hibridização com sondas moleculares. Revista da Sociedade Brasileira de Medicina Tropical (supl I):136,1995.

22. Passos VMA, Lasmar EB, Gontijo CMI. Fernandes O, Degrave W. Natural infection of domestic cat (Felis domesticus) with Ieishmaniti of the subgenus Viannia in the Metropolitan Region of Belo Horizonte. Memórias do Instiruto Oswaldo Cruz 89 (supl I):80, 1994.

23. Pedroso AM. Leishmaniose local do cão. Annaes Paulistas de Medicina e Cirurgia 1:33-39. 1913.

24. Pirmez C, Coutinho S, Marzochi MCA. Grimaldi $F^{\circ}$ G. Canine american cutaneous ieishmaniasis: a clinical and imunological study in dogs raturally infected with Leishmania braziliensis braziliensis in an endemic area of Rio de Janeiro, Brazil.The American Journal of Tropical Medicine and Hygiene 38:52-58, 1988.

25.Secretaria de Estado do Planejamento e Coordenação Geral (SEPLAN). Anuário Estatístico de Minas Gerais, Prodemge, Belo Horizonte, 1990.

26. Sessa PA, Falqueto A, Varejão JBM. Tentativa de controle da Leishmaniose Tegumentar Americana por meio do tratamento de cães doentes. Cadernos de Saúde Pública 10:457-463, 1994.

27. Simões-Barbosa F, Mello PA, Coura JR. Nota sobre a infecção natural de roedores por Leisbmania sp nos limites dos municípios de Teresópolis - Nova Friburgo, estado do Rio de Janeiro. Revista da Sociedade Brasileira de Medicina Tropical 4:113$115,1970$.

28. Snedecor GW, Cochran WG. The binomial distribution. In: Statistical methods, 7 th edition, University Press, Iowa p.107-130, 1980. 
Passos VMA, Andrade AC, Silva ES, Figueiredo EM, Falcão AL. Inquérito canino em foco recente de leisbmaniose tegumentar no município de Sabará, região metropolitana de Belo Horizonte. Revista da Sociedade Brasileira de Medicina Tropical 29:323-329, jul-ago, 1996.

29. Somarriba MMG, Valadares MG, Afonso MR. Conclusões. In: Lutas urbanas em Belo Horizonte. Editora Vozes, Petrópolis p. 119-125, 1984.

30. Yoshida, ELA, Correa FMA, Marques SA, Stolf HO, Dillon NL, Momem H, Grimaldi JR. Human, canine and equine (Equus cabalus) leishmaniasis due to Leishmania braziliensis in the south-west region of São Paulo State, Brazil. Memórias do Instituto Oswaldo Cruz 85:133-134, 1990. 